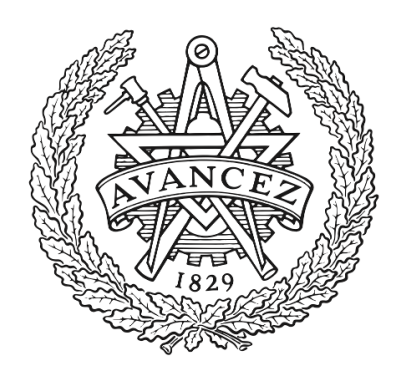

\title{
CHALMERS
}

UNIVERSITY OF TECHNOLOGY

\section{Multi-Panel Sparse Base Station Design with Physical Antenna Effects in Massive MU-MIMO}

Downloaded from: https://research.chalmers.se, 2023-04-26 09:24 UTC

Citation for the original published paper (version of record):

Amani, N., Wymeersch, H., Johannsen, U. et al (2020). Multi-Panel Sparse Base Station Design with Physical Antenna Effects in Massive MU-MIMO. IEEE Transactions on Vehicular Technology, 69(6): 6500-6510. http://dx.doi.org/10.1109/TVT.2020.2988612

N.B. When citing this work, cite the original published paper.

(O2020 IEEE. Personal use of this material is permitted.

However, permission to reprint/republish this material for advertising or promotional purposes 


\title{
Multi-Panel Sparse Base Station Design with Physical Antenna Effects in Massive MU-MIMO
}

\author{
Navid Amani, Henk Wymeersch, Ulf Johannsen, Adrianus Bart Smolders, Marianna V. Ivashina, and Rob \\ Maaskant
}

\begin{abstract}
A novel base station antenna (BSA) configuration is presented to mitigate degrading physical antenna effects in massive multiple-input multiple-output (MIMO) systems, while minimizing implementation complexities. Instead of using a commonly considered single antenna panel comprising of many elements covering a wide field-of-view (FOV) of 120 degrees, $L$ tilted panels are used employing $L$ times fewer elements and $L$ times smaller FOV per panel. The spatial resolution of each panel is enhanced by employing sparse arrays with suppressed (grating-lobe) radiation outside its corresponding FOV. Therefore, more directive antenna elements can be deployed in each panel to compensate for the effective isotropic radiated power (EIRP) reduction. While sectorisation reduces the antenna gain variation in 120 degrees FOV, cooperation among multiple panels in downlink beamforming is seen to be capable of interpanel interference suppression for sum-rate enhancement. A network model is used as a multi-user (MU) MIMO simulator incorporating both antenna and channel effects. It is shown that when the number of base station antennas is ten times the number of users, the average downlink sum-rate in pure line-of-sight (LOS), rich and poor multipath environments is increased up to $60.2 \%, 23 \%$ and $11.1 \%$, respectively, by multi-panel sparse arrays applying zero-forcing (ZF) precoding.
\end{abstract}

Index Terms-Base station antenna, beamforming, massive MIMO, mutual coupling, sparse array, zero-forcing.

\section{INTRODUCTION}

An ideal massive multiple-input multiple-output (MIMO) system for fifth generation $(5 \mathrm{G})$ wireless networks is capable of offering simultaneous improvement of spectral and energy efficiencies [1]. Orthogonal beams from an $M$-element base station antenna (BSA) will be able to provide service to $K \leq M$, user equipments (UEs) employing the same timefrequency resources. However, in a real scenario the number of antennas at the base station is limited. Furthermore, in physical propagation channels, which do not necessarily follow the theoretical independent and identically distributed (i.i.d.) Rayleigh fading channel model, the quality of service is influenced to a great extent by the level of interference [2], [3].

A real massive MIMO implementation is hindered by a high system complexity. As it is noted in [4], the routing of circuit components and integration of antenna elements become cumbersome within a single panel for large $M$.

N. Amani and R. Maaskant are with the Department of Electrical Engineering, Chalmers University of Technology, Gothenburg, Sweden, and also with the Electromagnetics Group, Eindhoven University of Technology (TU/e), The Netherlands (email: anavid@chalmers.se,n.amani@tue.nl).

H. Wymeersch and M. V. Ivashina are with the Department of Electrical Engineering, Chalmers University of Technology, Gothenburg, Sweden.

U. Johannsen and A. B. Smolders are with the Electromagnetics Group, Eindhoven University of Technology (TU/e), The Netherlands.
Cooling of the dissipated power turning into heat due to the low efficiency of the power amplifiers (PAs) in a dense array is challenging as well, in particular at mm-wave frequencies [5]. Regarding the latency of the system, the complexity of the perantenna and central signal processing algorithms grows with $M$ [6].

The idea of multi-panel massive MIMO as a $5 \mathrm{G}$ new radio (NR) access, especially for mm-wave frequency bands, was discussed in Third Generation Partnership Project (3GPP) meetings [7]. Research challenges and prospective future directions for multi-panel antenna structures are disclosed in [4]. Furthermore, some recent experimental evaluations of a multipanel massive MIMO configuration have revealed a significant throughput gain [8]. However, in their approach planar panels comprised of horizontal and vertical uniform linear arrays (ULAs) are deployed without any tilt angle among panels. That is, all antenna elements inside the multiple panels are contributing to a relatively wide field-of-view (FOV), e.g, $120^{\circ}$ in [8]. Although up to three sectors per site is known as a well-suited configuration for micro- and macro-cell base stations, a 6-sector BSA has been suggested for high capacity applications [9].

The most early studies on the performance of a Massive MIMO system are accomplished under common underlying assumptions of ideal or omni-directional radiators having negligible mutual coupling effects [1], [10]. Therefore, possible detrimental antenna effects on the system performance are underestimated. The physical antenna features which may degrade the system performance are: (i) element pattern tapering, which was shown to cause a sum-rate loss in the system when spatial multiplexing is employed [11], (ii) mutual coupling. The effect of mutual coupling is twofold: (ii-A) perturbation of the isolated element pattern (IEP), commonly known as embedded element pattern (EEP), (ii-B) mutual impedance, both due to the induced current on the neighboring elements by the radiated fields from the excited element [12].

A variety of studies have already appeared in the literature investigating the effect of mutual coupling on the MIMO system performance. While some works have shown that the mutual coupling can be useful for capacity enhancement [13], [14], others have reported it as a destructive phenomenon [11], [15], [16]. The reason behind these seemingly inconsistent conclusions is attributed to the different loading conditions (terminations) at the antenna ports. In [17], it is illustrated how various terminations can adjust the radiated power and therefore the diversity performance of coupled antennas. Paper [18] harvests the mutual coupling effect for sum-rate enhancement in a multiple-input single-output (MISO) system by optimizing 
the tunable loads connected to each antenna. Although the paper has stated that the proposed approach is suitable for slow or quasi-static fading channels, the implementation of a tunable load by means of a reconfigurable matching network is challenging as well.

While the physical antenna effects are carefully taken into account in the antenna community, the fading wireless channel, due to the non-line-of-sight (NLOS) propagation, is generally disregarded. Hence, a robust analysis of a MIMO system requires a model to incorporate both antenna and channel effects simultaneously [19], [20]. The circuit theory of communication can be applied for this purpose [21].

In this paper, a new structure for a $5 \mathrm{G}$ BSA is proposed to enhance the average downlink sum-rate of a multi-user (MU) MIMO system by mitigating the physical degrading antenna effects. The main contributions of this work can be summarized as three key components:

1) A novel 5G BSA configuration which deploys combined sectorization, panelization and sparsity. With the aid of the proposed structure, the performance of a MU-MIMO system exceeds that of a conventional ULA.

2) A generic network model of a MU-MIMO system which accounts for both antenna (mutual coupling, pattern tapering, polarization) and channel (multipath) effects. The proposed scheme enables us to examine the system performance in various channel models with different richness of multipath.

3) Demonstration of average sum-rate enhancement owing to the reduction of: (i) antenna gain variations by deploying multiple tilted panels; (ii) mutual coupling by introducing sparse arrays and consequently less severe mismatch losses, where antenna elements are terminated with a constant impedance.

Among the above-mentioned components, the generic network model can be considered as a general and stand-alone contribution. In terms of practicalities, the proposed modular scheme equipped with sparse arrays potentially facilitates the implementation challenges of a large antenna system.

This paper is organized as follows. A new BSA configuration, associated challenges and potential solutions, are presented in Section II. A generic network model is proposed in Section III as a simulation tool, capable of evaluating the performance of a MU-MIMO system, considering both antenna and channel effects. A flowchart of the co-simulation scenario is explained in this section as well. Section IV describes a case study with simulation results in order to make a comparison between the conventional single panel ULA and the proposed multi-panel sparse BSA configurations. Finally, conclusions are drawn in Section V.

Regarding the notation, boldface upper case and lower case denotes matrices and vectors, respectively, while field vectors are represented by overbar $(\bar{G})$. Transpose, complex-conjugate and Hermitian operators are denoted by $(.)^{T},(.)^{*}$ and $(.)^{\dagger}$, respectively. The expectation operator is denoted by $\mathbb{E}\{$.$\} .$

\section{Proposed BSA Configuration}

A tri-sector BSA is known as a standard configuration for macro-cell scenarios [9]. We consider this topology as a con-

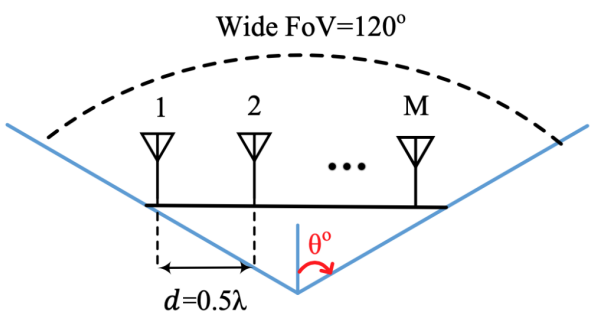

(a)

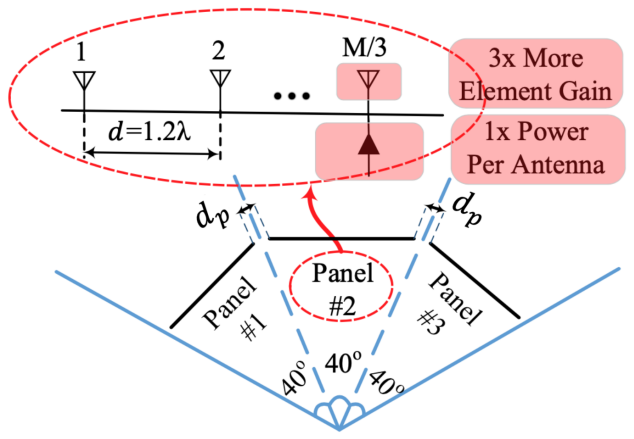

(b)

Fig. 1. One sector of a BSA covering a wide FOV of $120^{\circ}$ using (a) single panel conventional ULA with $d=0.5 \lambda$ spacing ( $\lambda$ represents the wavelength) and $M$ antennas; (b) multi-panel sparse array with $d=1.2 \lambda$ spacing and $M / 3$ number of antennas in each panel, where the total number of antennas is $M$.

ventional BSA with three sectors each covering a wide FOV of $120^{\circ}$. One sector is shown in Fig. 1(a). While BSA sectorisation has been found useful for capacity enhancement [22] and panelization for complexity reduction [4], combination of these two concepts eventuates in a practical solution for a massive MIMO BSA design.

Clustering the base station antenna elements into multiple tilted panels of sparse arrays, with a reduced FOV per panel, is utilized. The $M$ antennas in one sector of the conventional base station configuration can be equally divided into $L$ titled panels of sparse arrays (by increasing the inter-element spacing $d$ ) with a reduced FOV of $120^{\circ} / L$. Due to the smaller FOV per panel, antennas with higher gain and less gain variations inside the FOV can be used. Since multiple tilted panels cover a wide FOV $\left(120^{\circ}\right)$, the total gain pattern variation is the same as a single panel inside its corresponding FOV $\left(120^{\circ} / L\right)$. Furthermore, sparsity mitigates the effect of mutual coupling which consequently reduces the PA-antenna mismatch losses. These together increase the total radiated power by the BSA and accordingly improve the signal-to-interference-plus-noise ratio (SINR) at the UEs. Fig. 1(b) shows an example of the multi-panel configuration when $L=3$. A proper selection of $L$ depends on the antenna element radiation characteristics. The distance between multiple-panels $d_{p}$, in Fig. 1(b), should be selected in a way that the coupling effect among panels is minimal. The proposed modular approach moderates the degrading physical antenna effects and also facilitates the implementation complexities, however, certain drawbacks need to be overcome, as described below. 


\section{A. Challenges and Potential Solutions}

\section{1) Effective Isotropic Radiated Power (EIRP):}

Problem: In each single sparse array panel, the EIRP decreases due to the reduced number of antenna elements and their corresponding PAs. Furthermore, since panels in a multipanel configuration are tilted, not all antenna elements are effectively contributing to all UEs, e.g., the contribution of panel \#1 to the UEs inside the FOV of panel \#3 is minimal. Therefore, some preventative measures have to be taken in order to maintain the system performance.

Solution: Due to the limited FOV per panel, directive antenna elements with more gain can be deployed for the compensation of the EIRP reduction. In fact, since adjacent sparse array panels are tilted and having their own local broadside direction, typically with maximum element gain in this direction, the element gain enhancement can be even higher than $L \times$ for a range of angles in comparison with a conventional ULA shown in Fig. 1(a). For instance, panels \#1, $\# 2$ and \#3 in Fig. 1(b) are having their maximum element gain at $\theta=-40^{\circ}, \theta=0^{\circ}$, and $\theta=40^{\circ}$, respectively.

2) Spatial Resolution:

Problem: Another important antenna characteristic, especially when spatial multiplexing is applied, is the antenna spatial resolution. It is advantageous to have narrow beams since they are more capable of reducing the correlation between the channel vectors in a line-of-sight (LOS) situation. Even in a NLOS scenario, an antenna which has the capability of generating narrow beams offers more degrees of freedom for fine pattern shaping, although the effect of that depends on the channel state. For instance, this effect does not have an observable impact on the sum-rate under so-called favourable propagation, as defined in [10]. Reducing the number of elements in each panel makes the antenna aperture smaller and hence decreases the spatial resolution.

Solution: Sparse arrays with an increased inter-element spacing, rather than the standard $0.5 \lambda$ ( $\lambda$ is the wavelength) [23], can be utilized to enhance the spatial resolution. For the case of $L=3$, to attain the same spatial resolution by the sparse array panels, the inter-element spacing $d$ has to be increased by a factor of $L=3$, resulting in $d=1.5 \lambda$, to get the same aperture size. The larger spacing also facilitates the use of higher gain elements as discussed above. In addition, as it is shown in [14], [17] the effect of antenna termination on the capacity/diversity order, respectively, is becoming less important as the spacing between antennas increases. Therefore, using sparse arrays equipped with adaptive beamforming algorithms the effect of mutual coupling and consequently the PA-antenna mismatch losses are becoming less severe.

3) Grating Lobes:

Problem: The main concern of using sparse arrays, due to violating the Nyquist rate, is the appearance of grating lobes [24], [25]. In LOS, this may lead to highly correlated channel vectors even for large angular separation among users. This can occur if one UE is located at the direction of the grating lobe.

Solution: In [26] it is shown that, by employing a ULA equipped with a zero-forcing $(\mathrm{ZF})$ precoder, there exists a maximum allowable $d$ which prevents grating lobes from entering the FOV. For instance, $L=3$ results in $\mathrm{FOV}=40^{\circ}$ and by using [26, Eq. 18]:

$$
0.5 \lambda<d<1.46 \lambda \text {. }
$$

Grating lobes, even outside the FOV, have to be addressed properly in a multi-panel configuration since otherwise one panel can generate significant interferences for adjacent sectors. Since the radiation from a practical antenna element cannot drop immediately at the borders of the FOV, guard bands should be defined around it, within which the element pattern has to drop-off rapidly and sufficiently. Therefore, $d$ has to be selected in such a way that for any random distribution of UEs grating lobes do not appear, even inside the guard bands. The width of the guard bands depend on the actual element pattern and defines a trade-off between the grating lobe suppression and the antenna pattern spatial resolution.

\section{4) Inter-Panel Interference:}

Problem: Although the $\mathrm{ZF}$ precoding is an efficient approach for eliminating intra-panel interference, the interference among multiple panels, known as the inter-panel interference, has a detrimental effect on the system performance. In this case, the interference arriving at the UE terminals from the adjacent tilted panels degrades the UE's data rate dramatically. For instance, considering Fig. 1(b), the interference from panel $\# 1$ and \#3 to a UE inside the FOV of panel \#2 is a key data rate-limiting factor if the channel state information (CSI) of the adjacent sectors are not taken into account.

Solution: Although a single-cell MU-MIMO is investigated in this work, to address the above-mentioned problem we deploy the similarity between multi-panel and multi-cell scenarios. In a multi-panel configuration each panel is responsible to cover its corresponding sector. Therefore, sectors and panels in a multi-panel case resemble cells and base stations in a multi-cell scenario, respectively. Hence, the concept of MIMO cooperation [27], in a multi-cell scenario, triggers cooperative beamforming by multiple panels of sparse arrays in a multipanel configuration. In the proposed structure in Fig. 1(b), three tilted panels of sparse arrays are located alongside to cover a $120^{\circ}$ sector and they can all be connected to a central processor $(\mathrm{CP})$. The $\mathrm{CP}$ is responsible for multi-panel processing without the need for backhaul links. Therefore, beamforming can be done cooperatively by the three panels.

\section{NeTwORK MOdel OF A MU-MIMO SCENARIO}

In this section a unified MU-MIMO simulation platform, which incorporates both antenna and channel effects, is proposed using circuit theory of communication [21]. An equivalent network model of a MU-MIMO system in a downlink scenario is presented in Fig. 2. Assuming $M$ elements at the BSA and $K$ single-antenna UEs, the input/output voltage and current vectors are $\mathbf{v}_{1}=\left[v_{1,1}, \ldots, v_{1, M}\right]^{T}$, $\mathbf{i}_{1}=\left[i_{1,1}, \ldots, i_{1, M}\right]^{T}, \mathbf{v}_{2}=\left[v_{2,1}, \ldots, v_{2, K}\right]^{T}$, and $\mathbf{i}_{2}=$ $\left[i_{2,1}, \ldots, i_{2, K}\right]^{T}$. The model interrelates the transmitter and receiver port voltage/currents to one another by cascaded $\mathbf{Z}$ matrices. This $(M+K)$-port network model embeds a $K \times M$ 


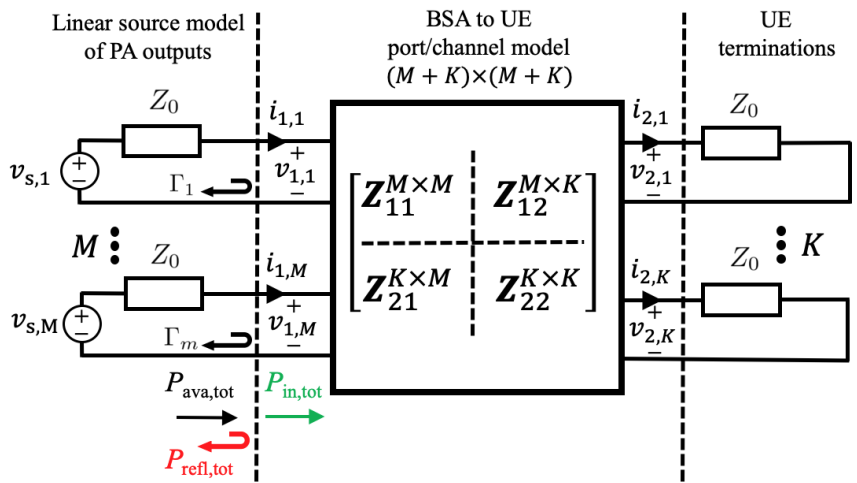

Fig. 2. Multiport network model of a MU-MIMO system including physical antenna effects.

channel matrix which is convenient for signal processing purposes. Readers are referred to [28] for a detailed explanation. The self-impedance matrix of the BSA $\mathbf{Z}_{11} \in \mathbb{C}^{M \times M}$, with off-diagonal elements representing the mutual impedance between BSA elements, can be computed from a full-wave simulation or a measurement campaign in the absence of the UEs. PAs are modeled as linear sources with a constant output impedance $Z_{0}$. The self-impedance matrix of the UEs, where off-diagonal elements account for the mutual coupling among them, is represented by $\mathbf{Z}_{22} \in \mathbb{C}^{K \times K}$.

\section{A. LOS and NLOS Channels}

The reaction concept between two antennas in the far-field is used to calculate the entries of $\mathbf{Z}_{12}$. We do this for a pure LOS channel and due to antenna reciprocity $\mathbf{Z}_{12}=\mathbf{Z}_{21}^{T}$. Their entries depend on the free-space impedance $\eta$, wavelength $\lambda$, and the LOS link distance $r_{k m}=\left\|\mathbf{r}_{k}-\mathbf{r}_{m}\right\|_{2}$ between the element $k$ and $m$. Furthermore, the normalized open-circuited EEP $\bar{G}^{\mathrm{oc}} / I$, where $I$ is the antenna excitation current, is also an affecting parameter as discussed in [25], i.e.,

$$
z_{12_{(m, k)}}=z_{21_{(k, m)}}=\frac{-2 j \lambda}{\eta}\left[\frac{\bar{G}_{k}^{\mathrm{oc}}\left(\hat{r}_{k}\right)}{I_{k}} \cdot \frac{\bar{G}_{m}^{\mathrm{oc}}\left(\hat{r}_{m}\right)}{I_{m}}\right] \frac{e^{-j \beta r_{k m}}}{r_{k m}},
$$

where $\beta$ is the wavenumber and $z_{12}$ represents the mutual impedance between a base station and UE antenna pair.

In a NLOS scenario, due to the absence of a direct path between the transmitter and the receiver, communication is possible via the scatterers. Traditionally, a uniform scattering environment, introduced by Clarke [29], is used to model the multipath in a wireless channel which results in a Rayleigh fading channel. This model might seem a highly idealized assumption since multipath components often arrive in clusters and the channel may deviate from a Rayleigh fading one [30], [31]. Furthermore, 5G mm-wave channel modeling based on extensive channel measurements eventuates in clusters of scatterers (CoSs) [32].

Although precise channel modeling is out of the scope of this paper, the network model has to be capable of evaluating the performance metric of a massive MIMO system in a generic NLOS environment. Therefore, we propose a Monte Carlo simulation within which random CoSs are defined in the

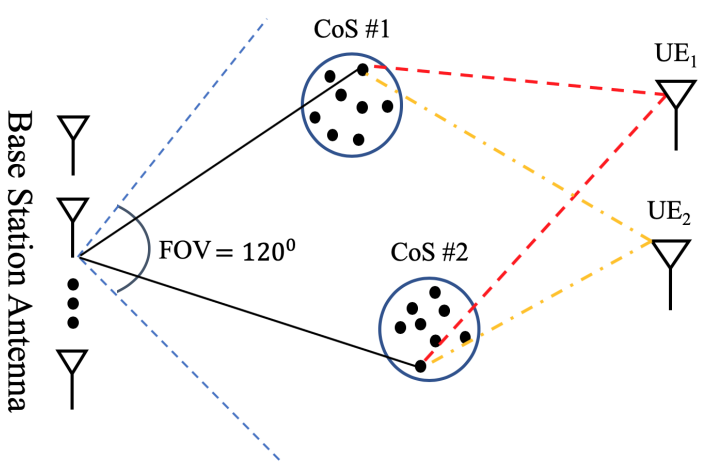

Fig. 3. NLOS channel model of a MU-MIMO system. Dashed blue lines shows the FOV of a sectorized BSA and black dots represent scatterers. Two UEs are randomly located inside the FOV.

environment, as shown in Fig. 3. The number of scatterers $(N)$ together with the number and density of CoSs are defining the richness of multipath in the NLOS environment, so that different channel models are realizable. The NLOS link distance is $r_{k n m}$ between the $k$-th user, $n$-th scatterer in the propagation channel and $m$-th element at the BSA. For the phase variation in the channel model, $r_{k n m}=r_{k n}+r_{n m}$ is used, while a transmission coefficient $T_{n}$ of each scatterer is considered for the amplitude variation [33]. Hence, in a NLOS scenario $\mathbf{Z}_{12}^{n}(n=1, \ldots, N)$ can be computed ${ }^{1}$. Afterwards, due to the linearity in the system, adding up the contribution of $N$ scatterers will include all the constructive and destructive field interference effects by the scatterers to yield $\mathbf{Z}_{12}=\sum_{n=1}^{N} \mathbf{Z}_{12}^{n}$ in the network model. The performance metric can be assessed in each simulation run and subsequently be analyzed in a statistical sense. It is pointed out that, under the assumption of narrowband communication, all the multipath components are almost arriving at the same time.

The $K \times M$ channel matrix $\mathbf{H}$, which interrelates the input and output voltage vectors $\mathbf{v}_{1}$ and $\mathbf{v}_{2}$, can be expressed in terms of the network parameters [28], i.e.,

$$
\begin{aligned}
\mathbf{H} & =\left(\mathbf{I}_{K}+\mathbf{Z}_{22} \mathbf{Z}_{l}^{-1}\right)^{-1} \mathbf{Z}_{21}\left(\mathbf{Z}_{11}-\mathbf{Z}_{21}^{T}\left(\mathbf{Z}_{l}+\mathbf{Z}_{22}\right)^{-1} \mathbf{Z}_{21}\right)^{-1} \\
& \approx\left(\mathbf{I}_{K}+\mathbf{Z}_{22} \mathbf{Z}_{l}^{-1}\right)^{-1} \mathbf{Z}_{21} \mathbf{Z}_{11}^{-1}
\end{aligned}
$$

where $\mathbf{I}_{K}$ is a $K \times K$ identity matrix and the diagonal matrix $\mathbf{Z}_{l}$ holds the UE load impedances.

Remark 1: Assuming that the mutual coupling effects between elements at the BSA and also between UEs are negligible, and that the input impedance of the antenna elements in isolation is considered to be $50 \Omega, \mathbf{Z}_{11}=50 \mathbf{I}_{M}$ and $\mathbf{Z}_{22}=50 \mathbf{I}_{K}$. Accordingly, the terms at both sides of $\mathbf{Z}_{21}$ in (3) can be removed as trivial factors. In addition, assuming omni-directional element patterns (another manifestation of ignoring mutual coupling effects to have equal IEPs) the trivial factors in (2) can also be omitted, and therefore the entries of the channel matrix are simply described by the term $\frac{1}{r_{k m}} e^{-j \beta r_{k m}}$. This forms the conventional LOS channel matrix in the wireless community [34].

\footnotetext{
${ }^{1}$ The cluster of scatterers could further represent an array of co-polarized and decoupled parallel metal wires each scattering the incident field omidirectionally in the plane of interest.
} 


\section{B. System Model and Co-Simulation}

We consider the downlink of a single-cell MU-MIMO system. The system, based on the network model presented in the preceding section, is represented as:

$$
\mathbf{v}_{2}=\sqrt{\alpha} \mathbf{H v}_{1}+\mathbf{u},
$$

where $\alpha$ is the normalization constant to adjust the transmit power and the UE receiver noise $\mathbf{u} \in \mathbb{C}^{K \times 1}$ satisfies a complex Gaussian distribution $\mathbf{u} \sim \mathcal{C} \mathcal{N}\left(\mathbf{0}, N_{0} \mathbf{I}_{K}\right)$, where $N_{0}$ is the noise power. The linearly precoded signal vector is

$$
\mathbf{v}_{1}=\mathbf{W} \mathbf{s},
$$

where $\mathbf{s}=\left[s_{1}, s_{2}, \ldots, s_{K}\right]^{T}, s_{k}$ representing a unit-power symbol intended for the $k$-th user, and $\mathbf{W} \in \mathbb{C}^{M \times K}$ is the precoding/weight matrix. We consider one symbol time slot in our system model. The weight matrix is the pseudo-inverse of the channel matrix $(\mathbf{H})$ by employing $\mathrm{ZF}$ precoding, i.e.,

$$
\mathbf{W}=\mathbf{H}^{\dagger}\left(\mathbf{H H}^{\dagger}\right)^{-1} \text {. }
$$

Precoding at the BSA, depending on the channel state, will lead to modified excitations at the antenna ports. Commonly in MU-MIMO studies, the total input power is normalized to have a constant value irrespective of the excitation vector [35]. However, from the antenna point of view changing the element's excitation results in varying mutual coupling effects. This deviates the input impedance of each antenna element from its isolated one. The impedance of an antenna element in an array when all elements are excited, is traditionally called active/scan impedance [25, Ch. 10]. This can significantly disturb the impedance matching condition between PAs and antennas which eventuates in mismatch losses. Due to this fact, the total input power will be smaller than the total available power $\left(P_{\text {in,tot }}<P_{\text {ava,tot }}\right)$ as shown in Fig. 2 . To examine the varying active impedance due to the adaptive beamforming, the normalization constant $\alpha$ in this paper is chosen to limit and equalize the total available power $\left(P_{\text {ava,tot }}\right)$ in all simulation scenarios.

From [28, Eq. (10)] and similar to (3)

$$
\mathbf{v}_{1}=\left(\mathbf{Z}_{11}-\mathbf{Z}_{21}^{T}\left(\mathbf{Z}_{l}+\mathbf{Z}_{22}\right)^{-1} \mathbf{Z}_{21}\right) \mathbf{i}_{1}=\mathbf{Z}_{\text {in }} \mathbf{i}_{1} \approx \mathbf{Z}_{11} \mathbf{i}_{1},
$$

where $\mathbf{Z}_{\text {in }}$ is the input impedance of the network model in Fig. 2. Therefore, the active impedance of the BSA elements $\left(Z_{\text {act }, m}\right)$ in the presence of the UEs and scatterers can be calculated by [25]

$$
Z_{\text {act }, m}=\frac{1}{I_{m}} \sum_{q=1}^{Q}\left[\mathbf{Z}_{\text {in }}\right]_{m, q} I_{q},
$$

where $\left[\mathbf{Z}_{\text {in }}\right]_{m, q}$ denotes the $(m, q)$-th element of the $\mathbf{Z}_{\text {in }}$ matrix. Afterwards, the active reflection coefficient at the antenna port $m$ when terminated with $Z_{0}$ is [36]

$$
\Gamma_{m}=\frac{Z_{\text {act }, m}-Z_{0}^{*}}{Z_{\text {act }, m}+Z_{0}} .
$$

Since practical antennas are commonly designed to attain $50 \Omega$ input impedance [24], the termination impedance $Z_{0}$ is also assumed to be $50 \Omega$. In order to incorporate the effect

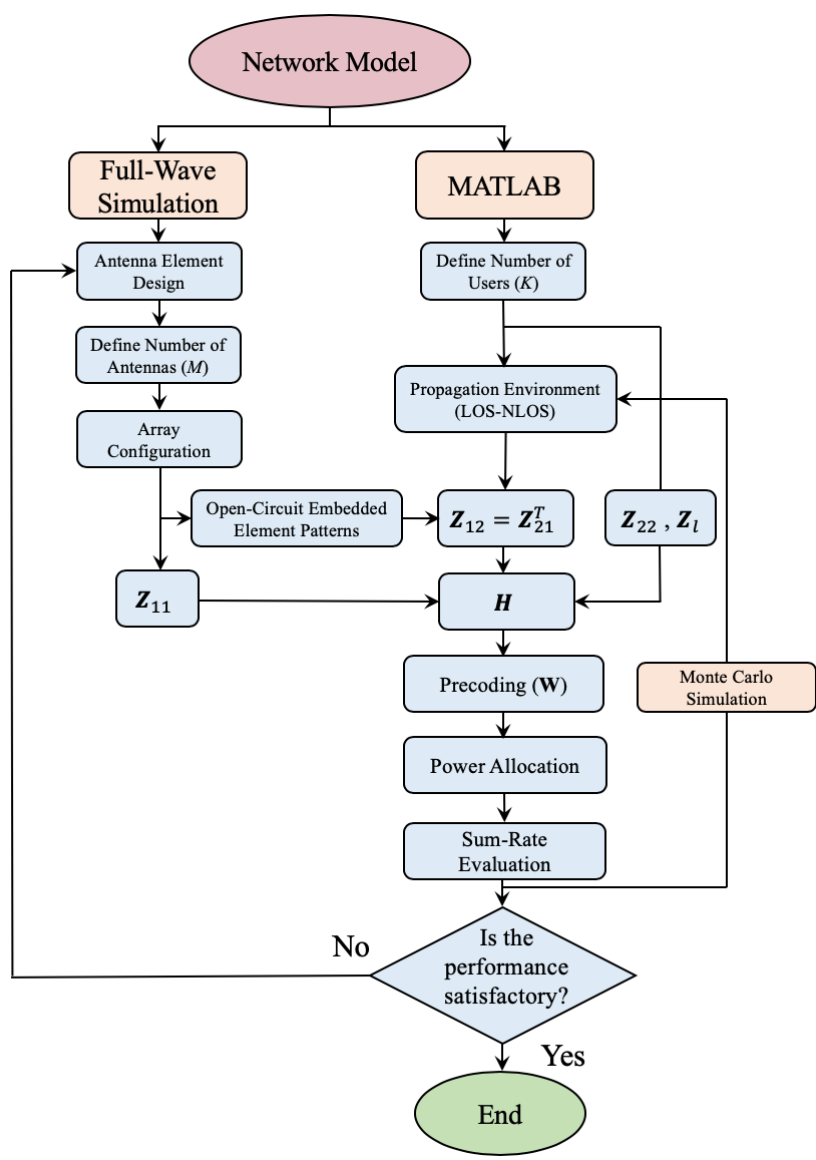

Fig. 4. A co-simulation flowchart of a MU-MIMO system for a BSA design.

of mismatch loss for different excitations, the decoupling efficiency $\eta_{\text {decoup }}$ can be used, which is defined as [37], [38]

$$
\eta_{\text {decoup }}=\frac{P_{\text {in }, \text { tot }}}{P_{\text {ava,tot }}}=\frac{\sum_{m=1}^{M} P_{\mathrm{acc}, m}}{\sum_{m=1}^{M} \frac{P_{\text {acc }, m}}{\left(1-\left|\Gamma_{m}\right|^{2}\right)}},
$$

where $P_{\mathrm{acc}, m}$ represents the accepted power at each antenna port $m$. To demonstrate the behaviour of $\eta_{\text {decoup }}$, an analytical example of the decoupling efficiency for the case of two short dipoles, modeled as Hertzian dipoles, can be found in Appendix A.

The total input power in each simulation run, using the approximation in (7), is ${ }^{2}$ :

$$
\begin{aligned}
P_{\text {in,tot }} & =\mathfrak{R e}\left\{\mathbf{i}_{1}^{\dagger} \mathbf{v}_{1}\right\}=\mathfrak{R e}\left\{\mathbf{v}_{1}^{\dagger}\left(\mathbf{Z}_{11}^{-1}\right)^{\dagger} \mathbf{v}_{1}\right\} \\
& =\mathfrak{R e}\left\{\mathbf{s}^{\dagger} \mathbf{W}^{\dagger}\left(\mathbf{Z}_{11}^{-1}\right)^{\dagger} \mathbf{W} \mathbf{s}\right\} \\
& =\frac{1}{2} \mathbf{s}^{\dagger} \mathbf{W}^{\dagger}\left(\mathbf{Z}_{11}^{-1}\right)^{\dagger} \mathbf{W} \mathbf{s}+\frac{1}{2} \mathbf{s}^{\dagger} \mathbf{W}^{\dagger}\left(\mathbf{Z}_{11}^{-1}\right) \mathbf{W} \mathbf{s}
\end{aligned}
$$

and considering $\mathbf{Z}_{11}$ as a symmetric matrix

$$
P_{\text {in,tot }}=\mathbf{s}^{\dagger} \mathbf{W}^{\dagger} \mathfrak{R e}\left\{\mathbf{Z}_{11}^{-1}\right\} \mathbf{W} \mathbf{s}
$$

\footnotetext{
${ }^{2}$ We have dropped the factor $1 / 2$ as this is customary in the array signal processing literature; RMS values are assumed.
} 
By taking (10) into consideration,

$$
P_{\text {ava,tot }}=\frac{\mathbf{s}^{\dagger} \mathbf{W}^{\dagger} \Re \mathfrak{R}\left\{\mathbf{Z}_{11}^{-1}\right\} \mathbf{W} \mathbf{s}}{\eta_{\text {decoup }}} .
$$

To fairly compare the performance of different BSA configurations in different simulation scenarios, the normalization constant $\alpha$ is defined as follows to have unity total available power, i.e.,

$$
\alpha=\frac{\eta_{\text {decoup }}}{\mathbf{s}^{\dagger} \mathbf{W}^{\dagger} \mathfrak{R e}\left\{\mathbf{Z}_{11}^{-1}\right\} \mathbf{W} \mathbf{s}} .
$$

In order to accomplish an accurate analysis of a MU-MIMO system including physical antenna effects, a co-simulation needs to be carried out. First, we select an antenna element and then define the array topology based on the element radiation characteristics and the number of elements forming the array. Since there are no closed-form expressions for the electric and magnetic fields in the near-field region of most practical antennas [24], we rely on a full-wave simulation, which numerically solves Maxwell's equations. The simulated $\mathbf{Z}_{11}$ matrix and open-circuited EEPs are imported in the MU-MIMO simulation platform where the propagation environment is defined for the end-performance analysis. Fig. 4 illustrates the cosimulation flowchart involving the full-wave simulator CST Microwave Studio [39], as well as MATLAB [40]. As shown in this figure, a Monte Carlo simulation is carried out to realize different channel states.

\section{Case Study and Simulation Results}

In this section, we first describe the implementation of a cosimulation platform (Fig. 4) using realistic antenna elements. We show how the physical antenna effects in a conventional BSA structure degrade the performance of a MU-MIMO system. Afterwards, we compare the performance of one sector of a conventional BSA and the proposed multi-panel sparse arrays configuration.

\section{A. MU-MIMO Simulator}

1) Antenna prototype: There are several beamformingenabled antenna designs for 5G applications based on patch antennas, at both mm-wave and sub $6 \mathrm{GHz}$ frequencies, [11], [41]-[45]. Herein, a single polarized patch antenna is selected to form a linear antenna array. The proposed concept can be readily extended to the orthogonal polarization and planar arrays. Layer \#1 in Fig. 5(a) represents a single polarized rectangular patch antenna, typically employed in conventional BSA configurations. Detailed design parameters are given in Appendix B. The patch antenna is tailored to achieve higher gain, required for the proposed multi-panel structure, by adding two layers of superstrates (layers \#2 and \#3). All layers have been designed and fabricated on a $0.75 \mathrm{~mm}$-thick Rogers RO3003 substrate.

As illustrated in Fig. 5(b), the superstrates reduce the antenna half-power beamwidth (HPBW) from $75^{\circ}$ to $36^{\circ}$. The element gain at the broadside direction increases from $7.18 \mathrm{dBi}$ to $12.2 \mathrm{dBi}$ - without increasing the occupied antenna area. By selecting $L=3\left(40^{\circ} \mathrm{FOV}\right.$ per sparse array panel), $8.4 \mathrm{~dB}$ gain variation of the single layer patch antenna

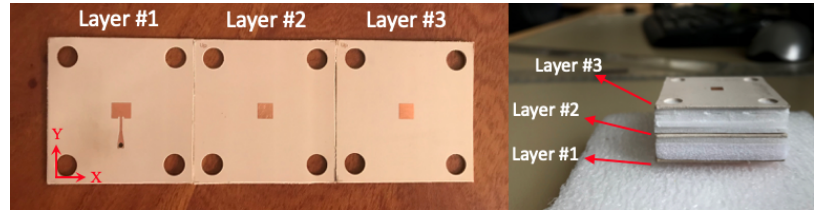

(a)

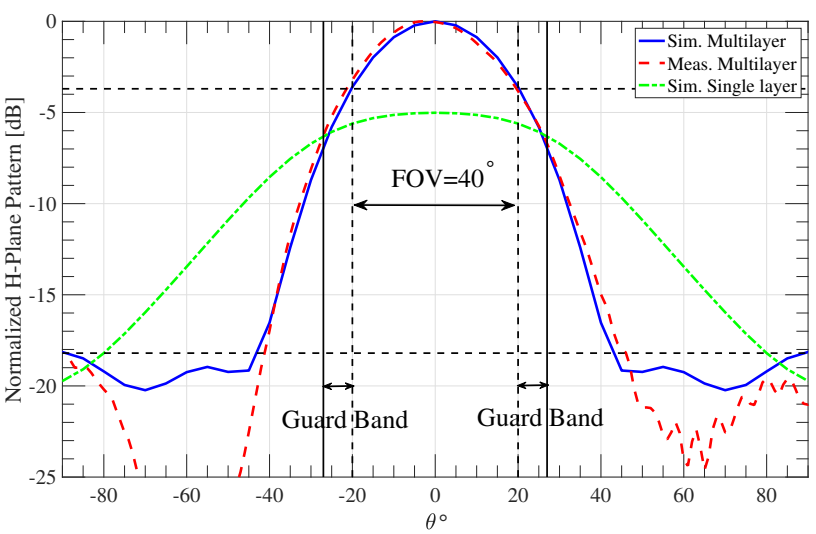

(b)

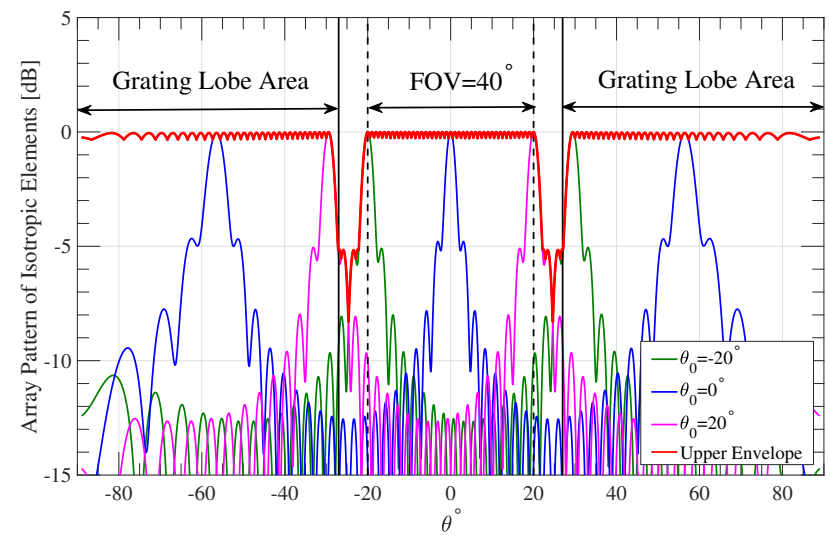

(c)

Fig. 5. (a) A fabricated multilayer-stacked patch antenna. Air-gaps between the substrate and superstrates are filled with foam layers, (b) simulated and measured normalized $\mathrm{H}$-plane radiation pattern of the proposed antenna element at resonance compared with its simulated counterpart from a single layer patch antenna (an H-plane linear array is in interest), (c) array patterns of a sparse array with $d=1.2 \lambda$ comprised of isotropic elements, when the main beam direction $\left(\theta_{0}\right)$ is located at $-20^{\circ}, 0^{\circ}$, and $20^{\circ}$. The red curve shows the upper envelop of several array patterns achieved by steering the main beam inside the $40^{\circ} \mathrm{FOV}$ with $1^{\circ}$ angular resolution.

in the $120^{\circ}$ FOV reduces to $3.6 \mathrm{~dB}$ by the multilayer-stacked patch antenna.

In order to prevent highly correlated UEs due to the appearance of grating lobes by sparse arrays, two guard bands, as shown in Fig. 5(b), are considered at both sides of the FOV within which the element radiation drops. Inside the guard bands, from $\pm 20^{\circ}$ to $\pm 27^{\circ}$, the radiation of the proposed multilayer-stacked patch antenna decreases by $3 \mathrm{~dB}$. Array patterns of isotropic radiators, when $d=1.2 \lambda$, are plotted in Fig. 5(c) for three directions $\left(\theta=-20^{\circ}, 0^{\circ}\right.$, and $\left.20^{\circ}\right)$. The mutual coupling between the elements in the array is neglected at this step. The appearance of grating lobes is obvious. Also, the upper envelope of several array patterns when the main beam is steered inside the $40^{\circ} \mathrm{FOV}$ with $1^{\circ}$ resolution is 
plotted. Based on Figs. 5(b) and (c), $d=1.2 \lambda$ does not allow the grating lobes to intrude into the guard bands. The unwanted radiation inside grating lobe areas is suppressed by the element pattern based on the pattern multiplication concept [24], [25].

2) Co-simulation: Full-wave simulations of the conventional ULA comprised of the single layer patch antennas and the sparse array formed by the multilayer-stacked patch antennas are conducted in CST Microwave Studio. Afterwards, the antenna impedance matrices and EEPs in the H-plane are extracted from CST and the Z-matrix blocks of the network model, as described in Section III, are calculated. The characteristics of the $\mathbf{Z}_{11}$ matrix of both arrays are discussed in Appendix B. The current-excited open-circuited EEP $\bar{G}_{m}$ of each array element is used in the reaction concept for the $\mathbf{Z}_{12}$ calculation, [cf. Eq. (2)]. In a pure LOS scenario $r_{k m}$ is used, while in a NLOS environment $r_{k n m}$ is utilized. For the sake of simplicity, omni-directional antennas are assumed at the receiver side. The on-diagonal elements of $\mathbf{Z}_{22}$ are equal to $50 \Omega$ and the off-diagonal elements are assumed zero.

For emulating the multipath in the simulation of a MUMIMO system we are interested in the qualitative performance figures and trends, also because there exists an uncertainty in the exact shape and material properties of the scatterers. Accordingly, $2^{4}$ scatterers are assumed to be spread in different number of CoSs, as shown in Fig. 3. The higher the transmission coefficients of scatterers, the stronger the multipath components. Herein, the transmission coefficient of $T_{n}=0.4$ is selected, as a representative value, for all scatterers ${ }^{3}$. Increasing the number of CoSs, can be interpreted as a realization of higher richness of multipath in the propagation environment [43], [46]. While $2^{1}$ CoSs represents a poor multipath environment, $2^{4} \mathrm{CoSs}$ mimics a relatively rich multipath effect where all scatterers are getting the complete freedom to be located anywhere in the environment.

\section{B. Physical Antenna Effects}

We will show how the physical patch antenna characteristics affect the system performance. To this end, the conventional ULA $(M=60, d=0.5 \lambda)$ is considered as one sector of the conventional BSA with $120^{\circ}$ FOV [see Fig. 1(a)].

1) Element Pattern Tapering: Assume a LOS channel with two UEs located at one Fraunhofer distance (FD) from the ULA at $\theta_{\mathrm{UE}_{1}}=0^{\circ}, \theta_{\mathrm{UE}_{2}}=-60^{\circ}$. While $\mathrm{UE}_{1}$ is standing at a fixed position (broadside of the ULA), the $\mathrm{UE}_{2}$ moves along a circular arc toward the $\mathrm{UE}_{1}$ till the angular separation between them becomes $0.5^{\circ}$. Computing the proposed channel matrix in (3) and applying the ZF precoder at the BSA, the acquired SINR at each UE terminal can be calculated similar to [35] as

$$
\operatorname{SINR}_{k}=\frac{\alpha\left|\mathbf{h}_{k}^{T} \mathbf{w}_{k}\right|^{2}}{\alpha \sum_{k^{\prime} \neq k}\left|\mathbf{h}_{k}^{T} \mathbf{w}_{k^{\prime}}\right|^{2}+N_{0}},
$$

where the noise power $N_{0}$ is selected in a way that a single user at one FD from a conventional ULA and at its broadside

\footnotetext{
${ }^{3}$ The exact scattering environment and thus the exact transmission coefficient of each scatterer toward the observer is irrelevant in the herein presented relative comparison study.
}

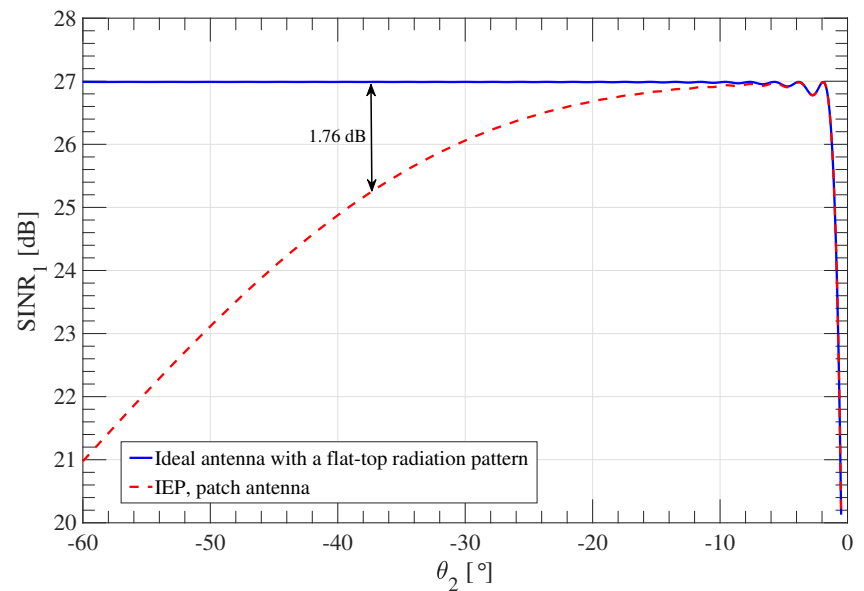

Fig. 6. SINR of the $\mathrm{UE}_{1}$ located at the broadside direction $\left(\theta_{\mathrm{UE}_{1}}=0^{\circ}\right)$ when $\mathrm{UE}_{2}$ moves from the left edge of the FOV toward the $\mathrm{UE}_{1},\left(-60^{\circ} \leq\right.$ $\left.\theta_{\mathrm{UE}_{2}} \leq-0.5^{\circ}\right)$.

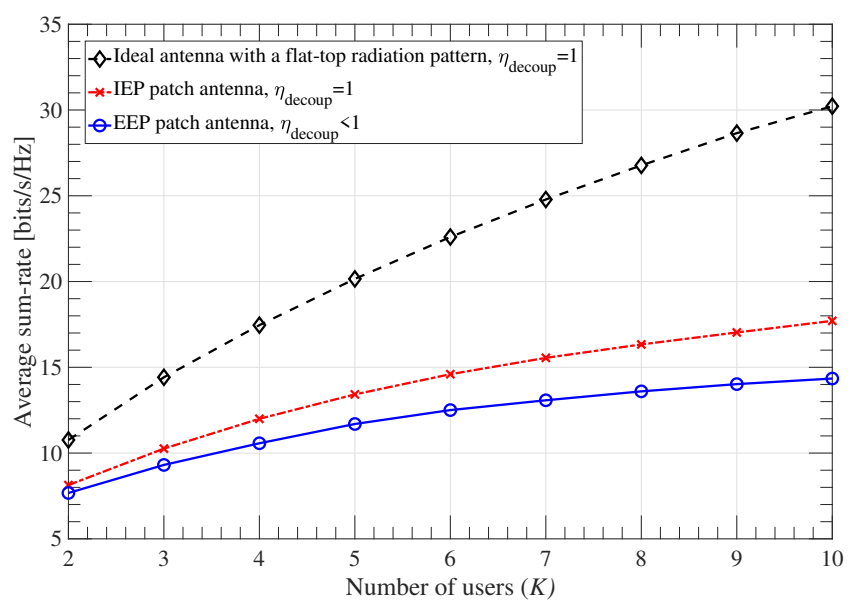

Fig. 7. Average sum-rate of a MU-MIMO system in a $120^{\circ} \mathrm{FOV}$, employing a ZF beamformed conventional ULA $(d=0.5 \lambda)$ in a LOS scenario.

direction attains a signal-to-noise ratio (SNR) of $30 \mathrm{~dB}$. The power allocation scheme at the BSA assures equal SINR per UE.

Fig. 6 compares the SINR of the first UE when ideal antennas with flat-top radiation pattern and the proposed single layer patch antennas are subsequently used at the base station. It can be seen that with ideal antennas, the $\mathrm{ZF}$ precoding mitigates the interference appropriately for large angular separations among two users. Since both UEs see the same element gain (regardless of their angle), the BSA allocates half of the power to each of them, hence $\mathrm{SINR}_{1}=27 \mathrm{~dB}$. The $\mathrm{SINR}_{1}$ drops significantly for very small angular separations since the channel vectors are becoming highly correlated in LOS. The same phenomenon is observed at small angular separations when patch antennas are deployed at the BSA. However, the $\mathrm{SINR}_{1}$ reduces gradually as the $\mathrm{UE}_{2}$ moves away from broadside. This happens due to tapering in the radiation pattern of the patch antenna (IEP is assumed here, [see Fig. 5(b)]). For instance, at $\theta_{\mathrm{UE}_{2}}=-37.5^{\circ}$ the isolated element gain drops by $3 \mathrm{~dB}$. In order to compensate this gain reduction 
at the direction of the second $\mathrm{UE}$, the allocated power to $\mathrm{UE}_{2}$ from the BSA is two times that of $\mathrm{UE}_{1}$. Therefore, two-third of the total input power goes to $\mathrm{UE}_{2}$ and consequently $\mathrm{UE}_{1}$ receives one-third of the input power. This reduces the $\mathrm{SINR}_{1}$ by $10 \log _{10}(3 / 2)=1.76 \mathrm{~dB}$ in comparison with the case of ideal antennas with a flat-top radiation pattern.

It should be noted that, the gain pattern variation is not restricted to patch antennas. In [11], it is shown that even dipole antennas, with an omni-directional radiation pattern in the H-plane, experience a significant embedded realized gain variation due to mutual coupling effects.

2) Mutual Coupling: In order to include mutual coupling effects at the BSA in a MU-MIMO system simulation, one needs to consider EEPs, instead of IEPs, as well as impedance mismatch effects. Since the excitation vector changes due to the adaptive beamforming, the average performance of the system is examined through a Monte Carlo simulation. The UEs are uniformly distributed inside the FOV within the range of one to five FDs from the BSA and with a minimum angular separation of $1^{\circ}$. The average sum-rate can be computed by [35]

$$
\mathrm{SR}=\sum_{k=1}^{K} \mathbb{E}\left\{\log _{2}\left(1+\mathrm{SINR}_{k}\right)\right\}
$$

where the expectation operation is taken over the random placement of multiple UEs in a LOS sceanrio.

The average sum-rate of the system versus the number of UEs is plotted in Fig. 7 using ideal and single layer patch antennas, with and without mutual coupling effects. In order to derive an achievable average sum-rate when physical antenna effects are disregarded, ideal antennas are considered. That is, radiators with flat-top radiation patterns inside the FOV and zero elsewhere are assumed, with maximum gain of the single layer patch antenna in the H-plane. Comparing the results with the case of using the IEP of the patch antenna without mutual coupling illustrates a significant sum-rate reduction due to the pattern tapering of the patch antenna. This can also be justified by Fig. 6. Next, we consider the effect of mutual coupling. To this end, EEPs are employed instead of IEPs and the decoupling efficiency is computed in each simulation run with the aid of the antenna active impedances. An observable degradation of the average sum-rate is seen to occur in Fig. 7 due to the reduced accepted power by the antenna elements as a consequence of the mismatch losses.

\section{Performance Comparison}

In this subsection, the average sum-rate using the proposed multi-panel configuration is compared with the one achievable by the conventional single panel ULA in different environments. By the titled arrangement of sparse array panels in the range of $\theta \in\left[-60^{\circ},-20^{\circ}\right]$ and $\theta \in\left[20^{\circ}, 60^{\circ}\right]$, a maximum element gain improvement of $9.8 \mathrm{~dB}$ is achieved. Meanwhile, the $8.4 \mathrm{~dB}$ gain variation inside $120^{\circ} \mathrm{FOV}$ reduces to 3.6 $\mathrm{dB}$. In addition, increasing the element spacing to $d=1.2 \lambda$ significantly reduces the mutual coupling degrading effects. The distance between multiple sparse panels is $d_{p}=4 \lambda$ since the effect of mutual coupling between multilayer-stacked patch

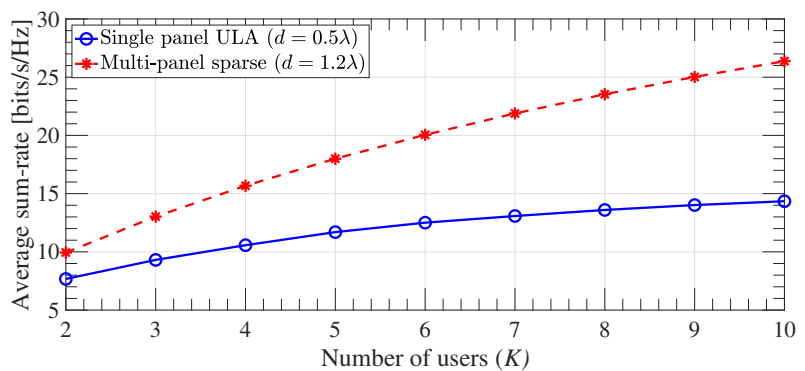

(a)

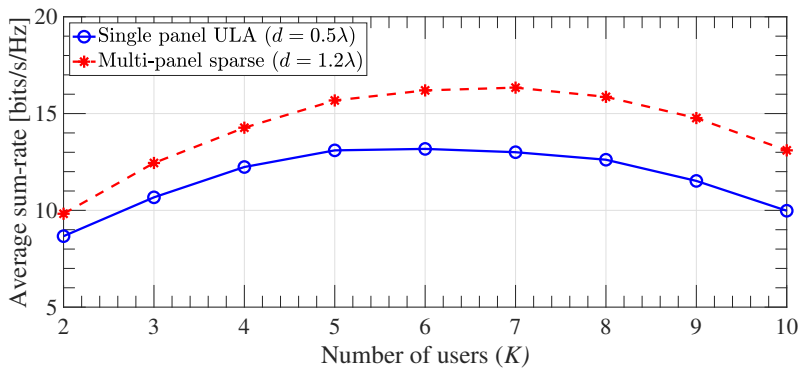

(b)

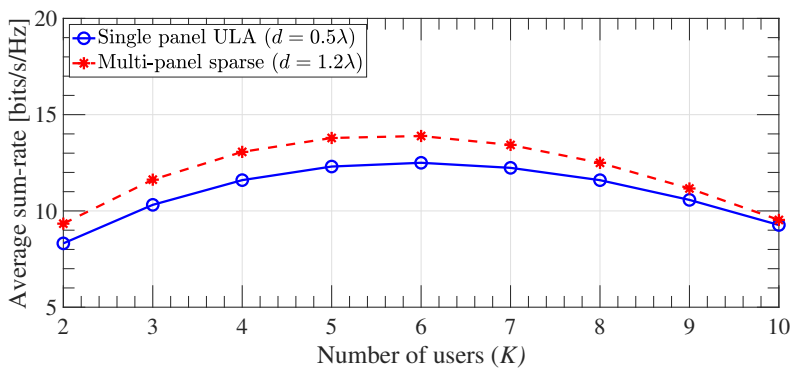

(c)

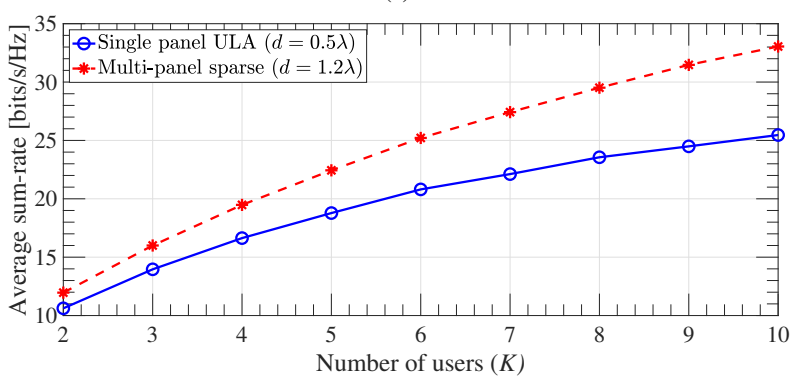

(d)

Fig. 8. Average sum-rate of a MU-MIMO system in a $120^{\circ} \mathrm{FOV}$, employing a ZF beamformed conventional ULA (comprised of single layer patch antennas) and multi-panel tilted sparse arrays (comprised of multilayer-stacked patch antennas) where $M=60$ in different environments: (a) LOS, (b) NLOS with $\# \mathrm{CoSs}=2^{4}$, (c) NLOS with \#CoSs $=2^{1}$, and (d) mixed LOS-NLOS with $\# \operatorname{CoSs}=2^{2}$.

antennas is seen to be negligible beyond this distance [cf. Appendix B]. In order to compare the performance of two BSA configurations, $10^{5}$ simulation runs are conducted in a Monte Carlo simulation. Both the UEs and the CoSs are randomly positioned inside the FOV assuming a uniform distribution. Changing the channel state adjusts the antenna excitations and consequently $\operatorname{SINR}_{k}$ in (15), hence the expectation in (16) is taken over random channel realizations. The average sum-rate versus the number of UEs is computed using (16) and plotted in Fig. 8 for different simulated environments. As 
can be observed in Fig. 8, the performance is improved by the multi-panel sparse array configuration in all representative environments.

Comparing the average sum-rate curve achieved by the multi-panel sparse configuration in pure LOS, plotted in Fig. 8(a), with the one obtained by ideal antenna elements in Fig. 7, confirms that the destructive physical antenna effects are considerably suppressed. While $55 \%$ of the achievable average sum-rate by ideal antennas is captured by the conventional ULA, for $K=6$ and $M / K=10$, this rate increases to $88 \%$ using the proposed multi-panel configuration. This corresponds to $60.2 \%$ enhancement of the average sum-rate by the proposed BSA structure in a pure LOS environment.

As illustrated in Fig. 8(b) and (c), the amount of improvement by the multi-panel configuration is less pronounced in NLOS compared to the pure LOS environments. This is due to the fact that among multiple scatterers in a NLOS scenario BSA exploits the ones which provide stronger signal paths to the UEs. Therefore, the element pattern tapering effect (gain variations) is a less degrading factor in multipath channels, although a rich multipath scattering eventuates in higher spatial variations and less correlation among UEs [43]. Hence, the amount of improvement in a NLOS scenario depends on the richness of multipath. Two representative NLOS scenarios in our simulation are selected as rich and poor multipath environments with $\# \mathrm{CoSs}=2^{4}$ and $\# \mathrm{CoSs}=2^{1}$, respectively. Fig. 8(b) and (c) show that the proposed multi-panel configuration improves the average sum-rate in these two NLOS environments by $23 \%$ and $11.1 \%$, respectively, compared to the conventional ULA when $M / K=10$. Moreover, for the cases that we have studied, the performance is also improved in mixed LOS and multipath environments, e.g., Fig. 8(d), with different number of CoSs and even different values of $T_{n}$.

It should be noted that, for the extraction of these results it is assumed that all three panels of sparse arrays are contributing to the downlink beamforming in all simulation scenarios, even for the case of $K=2$. However, clustering the antennas at the base station by simultaneous sectorization and panelization may bring other advantages. For instance, if there is not any significant signal path to one of the panels, this panel can decide to not contribute to the downlink beamforming without any noticeable performance degradation. In contrast, the effect of removing some antenna elements in a conventional ULA may not be negligible.

\section{CONCLUSION}

A new BSA configuration and its design requirements have been proposed for sum-rate enhancement of a MU-MIMO system by overcoming the performance degrading antenna effects. This has been accomplished by distributing $M$ antenna elements of one BSA sector over three tilted panels of sparse arrays each with a reduced FOV of $40^{\circ}$. The average downlink sum-rate has been evaluated by a network model in several propagation environments while accounting for physical antenna effects, such as antenna pattern tapering and mutual coupling effects. It has been illustrated that high gain antennas together with tilted panels reduce the antenna gain variations

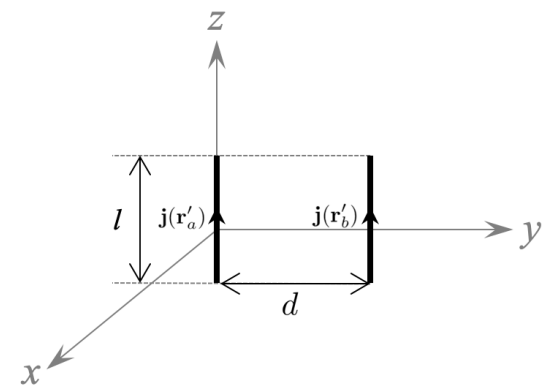

Fig. 9. Two short dipoles located side-by-side modeled as Hertzian dipoles.

inside the FOV. Furthermore, owing to the array sparsity, less severe mutual coupling effects and consequently reduced mismatch losses have been achieved. These together enhance the average downlink sum-rate by $11.1-60.2 \%$ depending on the propagation channels, when $M$ is ten times the number of UEs and the ZF precoding is applied to the BSA.

\section{APPENDIX A}

In order to analytically examine the concept of decoupling efficiency as a consequence of the mutual impedance, we consider two short dipoles $(l=0.001 \lambda)$, modeled as Hertzian dipoles, which are separated side-by-side by a distance $d$, as it is shown in Fig. 9. We assume that both dipoles are excited identically (with the same amplitude and phase). Since both dipoles are infinitesimally small, with respect to $\lambda$, the current distributions can be written as

$$
\mathbf{j}\left(\mathbf{r}_{p}^{\prime}\right)=I_{0} \delta\left(\mathbf{r}-\mathbf{r}_{p}^{\prime}\right) l \hat{z}, \quad p \in\{a, b\}
$$

where $\delta\left(\mathbf{r}^{\prime}\right)$ is the three-dimensional delta function and $\hat{z}$ is the unit vector. Computing the radiated electric field from the Hertzian dipoles using [25, Eq. (A.12)], the mutual impedance between them can be calculated by [25, Eq. (4.95)]. Since dipoles are $z$-oriented and located along the $y$-axis, using [25, Eqs. (4.34) and (C.26) $]^{4}, \hat{z} \cdot \hat{\mathbf{d}}=0$ and therefore the mutual impedance can be expressed as

$$
Z_{a b}=j \eta \beta l^{2}\left[1+\frac{1}{j \beta d}-\frac{1}{(\beta d)^{2}}\right] \frac{1}{4 \pi d} e^{-j \beta d} .
$$

We assume that each dipole antenna is terminated with the conjugate of its self-impedance $\left(Z_{a a}^{*}\right.$ and $\left.Z_{b b}^{*}\right)$. Hence, using (9)

$$
\Gamma=\frac{Z_{a b}}{2 R_{r}+Z_{a b}}
$$

where $R_{r}$ is the radiation resistance of a Hertzian dipole with a length $l$ in isolation [24, Eq. (4-19)]. Since a Hertzian dipole is a minimum scattering antenna when open-circuited, its self-impedance in the array remains the same as the one in isolation. Therefore, taking into account that both dipoles in Fig. 9 are excited identically, the decoupling efficiency is

$$
\eta_{\text {decoup }}=1-|\Gamma|^{2} \text {. }
$$

${ }^{4}$ For notation consistency, $\mathbf{R}$ in the reference [25] is replaced by $\mathbf{d}$ here. 


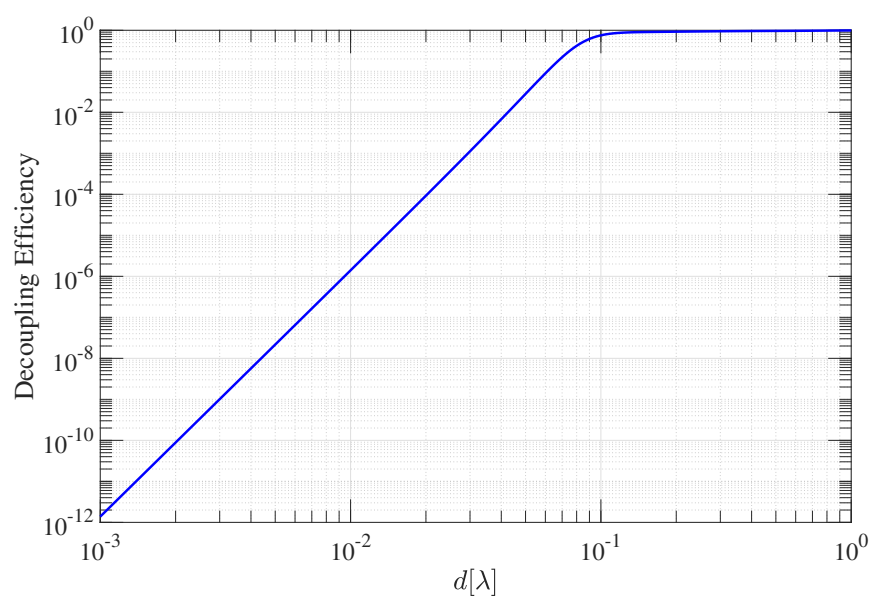

Fig. 10. Decoupling efficiency as a function of spacing between two identically excited short dipoles $(l=0.001 \lambda)$, modeled as Hertzian dipoles.

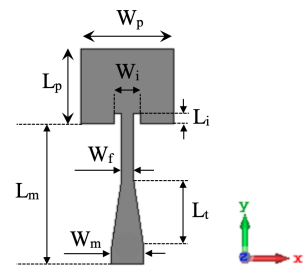

(a)

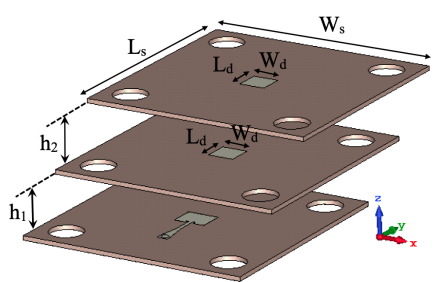

(b)
Fig. 11. (a) inset-fed microstrip patch antenna, (b) multilayer-stacked patch antenna. Dimensions in wavelengths are: $\mathrm{W}_{\mathrm{p}}=0.3283, \mathrm{~L}_{\mathrm{p}}=0.2652, \mathrm{~W}_{\mathrm{i}}=$ $0.0954, \mathrm{~L}_{\mathrm{i}}=0.036, \mathrm{~W}_{\mathrm{m}}=0.114, \mathrm{~L}_{\mathrm{m}}=0.4977, \mathrm{~W}_{\mathrm{f}}=0.0426, \mathrm{~L}_{\mathrm{t}}=0.24$, $\mathrm{W}_{\mathrm{s}}=\mathrm{L}_{\mathrm{s}}=2.4, \mathrm{~W}_{\mathrm{d}}=\mathrm{L}_{\mathrm{d}}=0.27, \mathrm{~h}_{1}=0.42, \mathrm{~h}_{2}=0.48$.

The decoupling efficiency as a function of distance $(d)$ between two short dipoles, which are excited uniformly, is plotted in Fig. 10 using (20). As it is illustrated, for a small separation the mutual impedance causes a significant mismatch loss and consequently a very low decoupling efficiency is observed. Considering the configuration in Fig. 9 as a transmit array, the reduced accepted power by the antennas for small separations decreases the signal level at the UEs, which adversely affects the achievable data rate.

\section{APPENDIX B}

Detailed design parameters of the inset-fed rectangular patch antenna, on a 0.75 mm-thick Rogers RO3003 substrate, are presented in Fig. 11(a). For reproducibility at any desired frequency, dimensions are given in terms of wavelength in the figure caption. This single layer patch antenna is deployed in the conventional single panel ULA with $0.5 \lambda$ spacing configuration. Adding two layers of superstrates on top of the proposed patch antenna forms the multilayer-stacked patch antenna, shown in Fig. 11 (b), for the sparse array panels where $d=1.2 \lambda$ is deployed.

The interaction between antenna elements, due to the mutual coupling, in both arrays are captured in their input impedance matrix $\left(\mathbf{Z}_{11}\right)$ through a full-wave simulation. For instance, self and mutual impedances of the edge element of both arrays,

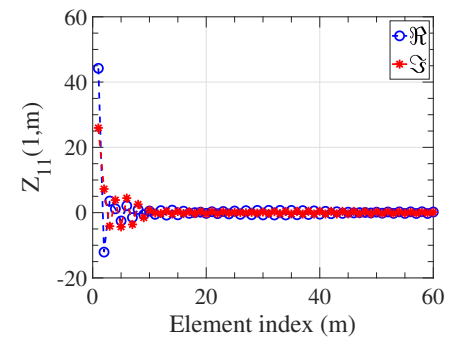

(a)

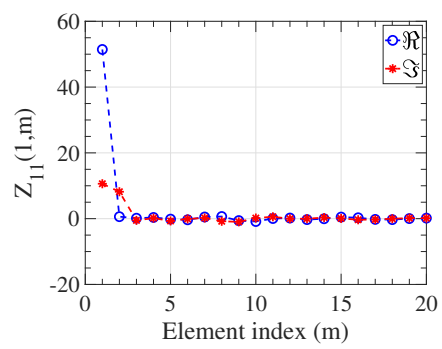

(b)
Fig. 12. Self and mutual impedance of the edge element in the (a) conventional ULA panel $(M=60, d=0.5 \lambda)$ comprised of a single layer patch antenna, (b) one sparse array panel $(M=20, d=1.2 \lambda)$ comprised of a multilayer-stacked patch antenna. Blue circles and red stars represent real and imaginary parts, respectively.

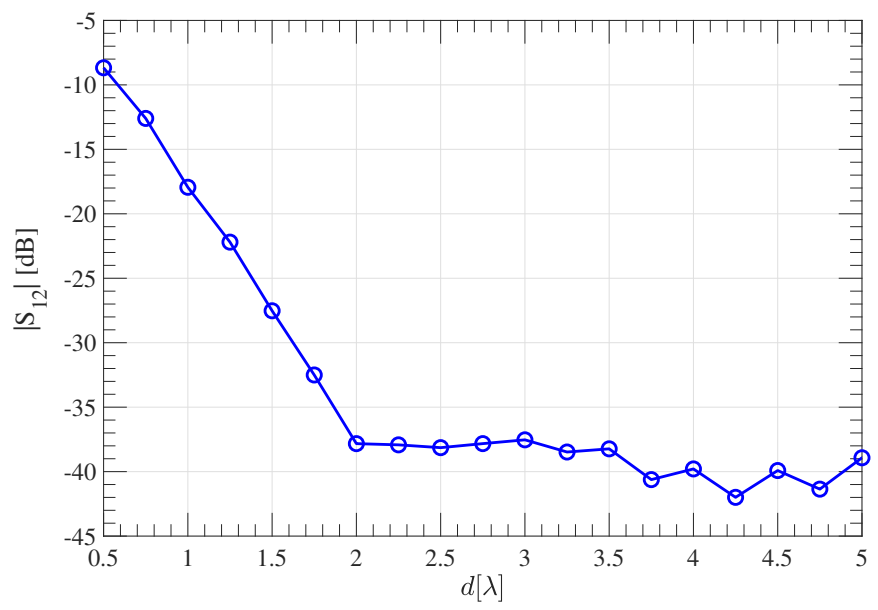

Fig. 13. Simulated $\left|S_{12}\right|$ between two multilayer-stacked patch antennas for different spacings $(d)$.

i.e., the conventional ULA $(M=60, d=0.5 \lambda)$ and one sparse array panel $(M=20, d=1.2 \lambda)$, are plotted in Fig. 12 . As can be observed, the real part of the self impedance in the sparse array is closer to $50 \Omega$, while the imaginary part is smaller than its counterpart in the conventional ULA. This can be interpreted as a better impedance matching condition in the sparse array. Furthermore, the mutual impedance decreases rapidly in the sparse array as the element index increases owing to the increased inter-element spacing. As mentioned in Section IV-C, the mutual interaction between two sparse array panels can be considered negligible for $4 \lambda$ spacing. In order to investigate this accurately, the isolation between two multilayer-stacked patch antennas for different spacings are extracted in terms of the scattering parameter $S_{12}$ through a full-wave simulation and plotted in Fig. 13. It is observed that an isolation around $40 \mathrm{~dB}$ is achieved when $d=4 \lambda$.

\section{ACKNOWLEDGMENT}

This project has received funding from the European Union's Horizon 2020 research and innovation programme under the Marie Skłodowska-Curie grant agreement No 721732.

The authors would also like to thank Dr. Bo Göransson and Prof. Christian Fager for their constructive comments. 


\section{REFERENCES}

[1] T. L. Marzetta, "Noncooperative cellular wireless with unlimited numbers of base station antennas," IEEE Trans. Wireless Commun., vol. 9, no. 11 , pp. 3590-3600, Nov. 2010.

[2] J. Flordelis, X. Gao, G. Dahman, F. Rusek, O. Edfors, and F. Tufvesson, "Spatial separation of closely-spaced users in measured massive multiuser MIMO channels," in Proc. IEEE Int. Conf. Commun. (ICC), Jun. 2015, pp. 1441-1446.

[3] C. M. Chen, V. Volskiy, A. Chiumento, L. Van der Perre, G. A. E. Vandenbosch, and S. Pollin, "Exploration of user separation capabilities by distributed large antenna arrays," in Proc. IEEE Global Commun. Conf. Workshops, Dec. 2016, pp. 1-6.

[4] Y. Huang, Y. Li, H. Ren, J. Lu, and W. Zhang, "Multi-panel MIMO in 5G," IEEE Commun. Mag., vol. 56, no. 3, pp. 56-61, Mar. 2018.

[5] Y. Aslan, J. Puskely, J. Janssen, M. Geurts, A. Roederer, and A. Yarovoy, "Thermal-aware synthesis of $5 \mathrm{G}$ base station antenna arrays: an overview and a sparsity-based approach," IEEE Access, vol. 6, pp. 58 868-58 882, Oct. 2018.

[6] L. Van der Perre, L. Liu, and E. G. Larsson, "Efficient DSP and circuit architectures for massive MIMO: State of the art and future directions," IEEE Trans. Signal Process., vol. 66, no. 18, pp. 4717-4736, Sep. 2018.

[7] H. Huawei, "Antenna structure: impact on MIMO transmission and remaining modeling issues," 3GPP TDocs Meet. id R1-86, Aug. 2016, [online]. Available: https://www.3gpp.org/DynaReport/TDocExMtg-R186-31663.htm.

[8] Z. Jiang, P. Chen, F. yang, and Q. Bi, "Experimental evaluation of a novel antenna structure: Multi-panel massive MIMO," in Proc. Int. Conf. Wireless Commun. Signal Process., Oct. 2018, pp. 1-5.

[9] J. Laiho, A. Wacker, and T. Novosad, Radio Network Planning and Optimisation for UMTS. Hoboken, NJ: Wiley, 2006.

[10] H. Q. Ngo, E. G. Larsson, and T. L. Marzetta, "Energy and spectral efficiency of very large multiuser MIMO systems," IEEE Trans. Commun., vol. 61, no. 4, pp. 1436-1449, Apr. 2013.

[11] C. M. Chen, V. Volski, L. Van der Perre, G. A. Vandenbosch, and S. Pollin, "Finite large antenna arrays for massive MIMO: characterization and system impact," IEEE Trans. Antennas Propag., vol. 65, no. 12, pp. 6712-6720, Dec. 2017.

[12] K. F. Warnick, R. Maaskant, M. V. Ivashina, D. B. Davidson, and B. D. Jeffs, Phased Arrays for Radio Astronomy, Remote Sensing, and Satellite Communications. UK: Cambridge University Press, 2018.

[13] T. Svantesson and A. Ranheim, "Mutual coupling effects on the capacity of multielement antenna systems," in Proc. IEEE Int. Conf. Acoust., Speech Signal Process., vol. 4, May 2001, pp. 2485-2488.

[14] J. W. Wallace and M. A. Jensen, "Mutual coupling in MIMO wireless systems: A rigorous network theory analysis," IEEE Trans. Wireless Commun., vol. 3, no. 4, pp. 1317-1325, Jul. 2004.

[15] R. Janaswamy, "Effect of element mutual coupling on the capacity of fixed length linear arrays," IEEE Antennas Wireless Propag. Lett., vol. 1, pp. $157-160,2002$.

[16] X. Chen, S. Zhang, and Q. Li, "A review of mutual coupling in MIMO systems," IEEE Access, vol. 6, pp. 24 706-24 719, 2018.

[17] J. W. Wallace and M. A. Jensen, "Termination-dependent diversity performance of coupled antennas: Network theory analysis," IEEE Trans. Antennas Propag., vol. 52, no. 1, pp. 98-105, Jan. 2004.

[18] A. Li, C. Masouros, and M. Sellathurai, "Analog-digital beamforming in the MU-MISO downlink by use of tunable antenna loads," IEEE Trans. Veh. Technol., vol. 67, no. 4, pp. 3114-3129, Apr. 2018.

[19] J. Gong, J. F. Hayes, and M. R. Soleymani, "The effect of antenna physics on fading correlation and the capacity of multielement antenna systems," IEEE Trans. Veh. Technol., vol. 56, no. 4, pp. 1591-1599, Jul. 2007.

[20] R. Bhagavatula, C. Oestges, and R. W. Heath, "A new double-directional channel model including antenna patterns, array orientation, and depolarization," IEEE Trans. Veh. Technol., vol. 59, no. 5, pp. 2219-2231, Jun. 2010

[21] M. T. Ivrlac and J. A. Nossek, "Toward a circuit theory of communication," IEEE Trans. Circuits Syst. I, Regul. Pap., vol. 57, no. 7, pp. 1663-1683, Jul. 2010.

[22] A. Wacker, J. Laiho-Steffens, K. Sipila, and K. Heiska, "The impact of the base station sectorisation on WCDMA radio network performance," in Proc. IEEE Veh. Technol. Conf., vol. 5, Sep. 1999, pp. 2611-2615.

[23] H. Krim and M. Viberg, "Two decades of array signal processing research," IEEE Signal Processing Mag., vol. 13, no. 4, pp. 67-94, Jul. 1996.

[24] C. A. Balanis, Antenna Theory: Analysis and Design. New York, NY, USA: Wiley, 2016.
[25] P.-S. Kildal, Foundations of Antenna Engineering: A Unified Approach for Line-of-Sight and Multipath. Artech House, 2015.

[26] N. Amani, A. A. Glazunov, M. V. Ivashina, and R. Maaskant, "Perantenna power distribution of a zero-forcing beamformed ULA in pure LOS MU-MIMO," IEEE Commun. Lett., vol. 22, no. 12, pp. 2515-2518, Dec. 2018.

[27] D. Gesbert, S. Hanly, H. Huang, S. S. Shitz, O. Simeone, and W. Yu, "Multi-cell MIMO cooperative networks: A new look at interference," IEEE J. Sel. Areas in Commun., vol. 28, no. 9, pp. 1380-1408, Dec. 2010.

[28] N. Amani, R. Maaskant, A. A. Glazunov, and M. Ivashina, "Network model of a 5G MIMO base station antenna in a downlink multi-user scenario," in Proc. 12th Eur. Conf. Antennas Propag. (EuCAP), Apr. 2018, pp. $1-5$.

[29] R. Clarke, "A statistical theory of mobile-radio reception," Bell Syst. Tech. J., vol. 47, no. 6, pp. 957-1000, 1968.

[30] J. M. Meredith, "Spatial channel model for multiple input multiple output (MIMO) simulations," 3GPP TR 25.996, 2012, [online]. Available: https://portal.3gpp.org/desktopmodules/Specifications/SpecificationDetails.aspx ?specificationId $=1382$.

[31] J. Meinilä, P. Kyösti, T. Jämsä, and L. Hentilä, "WINNER II channel models," Radio Technologies and Concepts for IMT-Advanced, pp. 3992, 2009.

[32] M. Peter, K. Haneda, S. L. H. Nguyen, A. Karttunen, J. Järveläinen, A. Bamba, R. D' Errico, J. Medbo, U. F S. Jaeckel, I. N, J. Luo, M. Rybakowski, C. Diakhate, J. Conrat, A. Naehring, S. Wu, A. Goulianos, and E. Mellios, "Measurement results and final mmMAGIC channel models," Deliverable D2.2, May. 2017. [Online]. Available: [online]. Available:https://bscw.5gmmmagic.eu/pub/bscw.cgi/d202656/mmMAGIC_D2_2.pdf

[33] M. E. Bialkowski, P. Uthansakul, K. Bialkowski, and S. Durrani, "Investigating the performance of MIMO systems from an electromagnetic perspective," Microw. Opt. Tech. Lett., vol. 48, no. 7, pp. 1233-1238, Jul. 2006.

[34] D. Tse and P. Viswanath, Fundamentals of Wireless Communication. Cambridge University Press, 2005.

[35] H. Q. Ngo, Massive MIMO: Fundamentals and system designs. Linköping University Electronic Press, 2015, vol. 1642.

[36] K. Kurokawa, "Power waves and the scattering matrix," IEEE Trans. Mircrow. Theory Tech., vol. 13, no. 2, pp. 194-202, Mar. 1965.

[37] M. N. M. Kehn, M. V. Ivashina, P.-S. Kildal, and R. Maaskant, "Definition of unifying decoupling efficiency of different array antennas-case study of dense focal plane array feed for parabolic reflector," Int. J. Electron. Commun., vol. 64, no. 5, pp. 403-412, May 2010.

[38] M. V. Ivashina, M. N. M. Kehn, P.-S. Kildal, and R. Maaskant, "Decoupling efficiency of a wideband vivaldi focal plane array feeding a reflector antenna," IEEE Trans. Antennas Propag., vol. 57, no. 2, pp. 373-382, Feb. 2009.

[39] Computer Simulation Technology, "CST Microwave Studio 2018," https://www.cst.com/, 2018.

[40] Mathworks, "MATLAB 2018," https://www.mathworks.com/, 2018.

[41] K. Kibaroglu, M. Sayginer, T. Phelps, and G. M. Rebeiz, "A 64-element $28-\mathrm{GHz}$ phased-array transceiver with 52-dBm EIRP and 8-12-Gb/s 5G link at 300 meters without any calibration," IEEE Trans. Microw. Theory Tech., vol. 66, no. 12, pp. 5796-5811, Dec. 2018.

[42] A. van den Biggelaar, U. Johannsen, M. Geurts, and A. Smolders, "Assessment on the frequency dependent performance of active phased arrays for 5g," in Proc. 13th Eur. Conf. Antennas Propag. (EuCAP), Mar./Apr. 2019, pp. 1-4.

[43] X. Gao, O. Edfors, F. Rusek, and F. Tufvesson, "Massive MIMO performance evaluation based on measured propagation data," IEEE Trans. Wireless Commun., vol. 14, no. 7, pp. 3899-3911, Jul. 2015.

[44] P. Xingdong, H. Wei, Y. Tianyang, and L. Linsheng, "Design and implementation of an active multibeam antenna system with $64 \mathrm{RF}$ channels and 256 antenna elements for massive MIMO application in 5G wireless communications," China Commun., vol. 11, no. 11, pp. 16-23, Nov. 2014.

[45] P. Harris, S. Malkowsky, J. Vieira, E. Bengtsson, F. Tufvesson, W. B. Hasan, L. Liu, M. Beach, S. Armour, and O. Edfors, "Performance characterization of a real-time massive MIMO system with LOS mobile channels," IEEE J. Sel. Areas in Commun., vol. 35, no. 6, pp. 12441253, Jun. 2017.

[46] P.-S. Kildal and J. Carlsson, "New approach to OTA testing: RIMP and pure-LOS reference environments \& a hypothesis," in Proc. 7th Eur. Conf. Antennas Propag. (EuCAP), Apr. 2013, pp. 315-318. 